\title{
Inhibición de Streptococcus mutans aislado de cavidad oral de niños sin caries mediante sustancia antagónica producida por Lactobacillus spp.
}

Willy Bustillos Torrez, ${ }^{1}$ Zulema Susy Bueno Bravo. ${ }^{2}$

\section{Resumen}

La caries es considerada todavía una de las enfermedades más prevalentes del mundo. Por este motivo, diversas estrategias de prevención se han desarrollado en los últimos años con el propósito de controlar esta enfermedad. Diferentes especies bacterianas de la microbiota normal de la cavidad oral producen sustancias antagónicas antibacterianas. Objetivo: El objetivo de este trabajo fue identificar alguna especie del género Lactobacillus productor de sustancias antagónicas contra Streptococcus mutans a partir de saliva de niños con y sin caries. Materiales y métodos: Se aislaron desde saliva de 60 niños con caries, sin caries activa (rehabilitados) y libres de caries, diferentes cepas de Lactobacillus a las cuales se les estudió su capacidad antagónica contra cepas de Streptococcus mutans, mediante ensayos en doble capa, test del pocillo y sobre crecimiento bacteriano. Resultados: Las cepas que elaboran sustancias con mayor capacidad antagónica fueron identificadas como Lactobacillus fermentum mediante Api test $50 \mathrm{CH}$. Conclusión: Se demostró que Lactobacillus fermentum está presente en mayor porcentaje en el grupo de niños sin caries, lo cual podría sugerir un efecto natural de control biológico en la cavidad oral de este grupo de niños.

Palabras clave: Lactobacillus, caries dental, inhibición, Streptococcus mutans.

1 Magister en Ciencias Biomédicas mención Microbiologia Clínica (tesista), Universidad de Talca. Chile.

${ }^{2}$ Especialista en Odontopediatria, Universidad Mayor de San Andrés, La Paz, Bolivia. 
Artigo original

\title{
Inibição do Streptococcus mutans isolado da cavidade oral de crianças sem cárie por substância antagônica produzida por Lactobacillus spp.
}

\section{Resumo}

A cárie dentária ainda é considerada uma das doenças mais prevalentes no mundo. Por esta razão, várias estratégias de prevenção foram desenvolvidas nos últimos anos com o objetivo de controlar esta doença. Diferentes espécies bacterianas da microbiota normal da cavidade oral produzem substâncias antagonistas antibacterianas. Objetivo: O objetivo deste estudo foi identificar algumas espécies do gênero Lactobacillus produtoras de substâncias antagonistas contra Streptococcus mutans da saliva de crianças com e sem cárie. Materiais e Métodos: Foram isolados a partir da saliva de 60 crianças com cavidades, sem cavidades ativas (restaurada) e livre de lesões de cárie, diferentes tipos de Lactobacillus que foram estudados pela sua capacidade antagonista contra Streptococcus mutans, por testes camada dupla ebem sobreocrescimentobacteriano. Resultados: As cepas que elaboraram substâncias com maior capacidade antagônica foram identificadas como Lactobacillus fermentum pelo teste Api 50 $\mathrm{CH}$. Conclusões: Foi demonstrado que o Lactobacillus fermentum está presente em maior porcentagem no grupo de crianças sem cárie, o que poderia sugerir um efeito natural do controle biológico na cavidade bucal desse grupo de crianças.

Palavras chave: Lactobacillus, cárie, inibição, Streptococcus mutans.

Original article

\section{Streptococcus mutans inhibition by antagonistic substance produced by Lactobacillus spp isolated from oral cavity in children without caries.}

\begin{abstract}
Caries is still considered one of the most prevalent diseases worldwide. For this reason, in recent years various prevention strategies have been developed with the purpose of managing this disease. Different oral cavity bacterial species part of the normal microbiota produce antibacterial antagonistic substances. Objective: The objective of this study was to identify

some species of Lactobacillus genus that produce antagonistic substances against Streptococcus mutans from saliva of children with and without caries. Materials and methods: Different Lactobacillus strains were isolated from saliva of 60 children with caries, without active decay (rehabilitated) and free of caries. The antagonistic capacity against strains of Streptococcus mutans was studied, by means of tests in double layer, test of the well and about bacterial growth.
\end{abstract}


Results: Lactobacillus strains that produced substances with greater antagonistic capacity were identified as Lactobacillus fermentum by Api test $50 \mathrm{CH}$. Conclusions: It was found that Lactobacillus fermentum is present in a larger percentage among

\section{Introducción}

En 2012, la Organización Mundial de la Salud (OMS) declaro que entre el 60\% y el $90 \%$ de los niños y casi el $100 \%$ de los adultos presentan caries. ${ }^{1}$

En los últimos años, ha habido una disminución en la prevalencia de caries dentales, especialmente en los países desarrollados, pero sigue siendo uno de los problemas de salud pública mundial más importantes que afectan a los niños. ${ }^{1}$ Una definición de "caries dental es causada por la disbiosis patobiontes", dejando de lado el concepto de enfermedad infecciosa y manteniendo su origen multifactorial, ${ }^{2}$ recientemente postulado. Varias estrategias de control siempre han implementado un enfoque de precaución, ya que los agentes de quimioprofilaxis como la clorhexidina, el trieclosán y los compuestos derivados de las plantas, eventualmente llevan al desarrollo de vacunas y al uso de probióticos. ${ }^{3}$

La visión ecológica que prevalece en la etiología de la caries dental es polimicrobiana por la constitución de la naturaleza del biofilm oral, sin embargo, se sabe que ciertas bacterias son aisladas de las lesiones cariosas constantemente, como son: Lactobacillus spp. y Streptococcus mutans ambas asociadas con caries dental. ${ }^{4}$ Así, en 1900, Lactobacillus fue el primer organismo conocido que causa caries en children without caries which could suggest a natural biological control effect by this bacterial strain.

Key words: Lactobacillus, Dental caries, Inhibition, Streptococcus mutans.

los dientes antes que Streptococcus mutans se describiera en las pruebas con ratas. ${ }^{5}$ Pero a pesar de los muchos estudios que relacionan Lactobacillus con caries en niños y adultos, la comprensión de su papel basado en el presente y otros estudios abre un debate sobre su papel en la presencia de salud o enfermedad. ${ }^{6}$

Streptococcus mutans es la bacteria oral más cariogénica más estudiada y reconocida. ${ }^{7}$

Existe evidencia científica de que el género Lactobacillus tiene un rol protector basado en la producción de diferentes tipos de bacteriocinas, sustancias proteínicas con diferentes mecanismos de acción antibacteriana. ${ }^{8}$ Algo similar ocurre con Streptococcus, especies conocidas y nuevas como Streptococcus dentisani que prometen ser una nueva alternativa terapéutica a la caries dental. ${ }^{9}$ Además, se ha observado que los probióticos presentan diversas propiedades, antagónicas en diferentes especies bacterianas, a través de la producción de bacteriocinas que contribuyen a la exclusión competitiva a través del nicho de ganancias para evitar la adhesión de patógenos. ${ }^{10}$

Se sabe que Lactobacillus spp. pertenecen a la microbiota oral y su capacidad para producir bacteriocinas exhibe una capacidad adhesiva significativa, y estas cepas también pueden reducir la cantidad de Streptococcus mutans de la superficie 
del diente cubierta por saliva. ${ }^{11}$ Una de las especies con mayor capacidad productiva de sustancias antagónicas es Lactobacillus paracasei, que incluye la inhibición de Streptococcus mutans, también bacterias periodontopáticas e incluso hongos. ${ }^{12}$ Hay alrededor de 10 especies de Lactobacillus orales presentes en las lesiones cariosas, y cohabitan con otros. Lactobacillus fermentum, Lactobacillus casei/paracasei y Lactobacillus salivarius siempre se encuentran en estado planctónico. ${ }^{13}$

Estudios recientes en niños muestran una correlación entre la presencia de Lactobacillus en la cavidad bucal y la caries dental, pero también en los niños con pocas caries. ${ }^{14}$ Otro estudio muestra la presencia de Lactobacillus en fisuras oclusales de niños sin caries. ${ }^{15}$ También en algunas caries infantiles se ha identificado en sus respectivas biopelículas una cantidad significativa de Lactobacillus. ${ }^{16}$

El propósito de este estudio fue determinar la presencia de cepas de Lactobacillus en la saliva de niños con caries, sin caries y caries inactivas haciendo una correlación además con sus respectivos riesgo de caries, los cuales fueron determinados mediante la herramienta CAST modificada (caries assessment spectrum and treatment), nuestra hipótesis es la producción de sustancias antagónicas (bacteriocinas) contra cepas de Streptococcus mutans.

\section{Material y métodos}

Los sujetos de estudio fueron seleccionados por conveniencia de la Escuela Prosperidad de Talca, establecimiento con un índice de vulnerabilidad del 95\%, ubicado en la ciudad de Talca entre la Calle 12 con 6 Oriente. Cuenta con más de 100 años de historia y sirve en kindergarten (preescolar) y educación básica hasta el $8^{\mathrm{o}}$ grado. La matrícula en 2015 ascendió a 350 estudiantes por gestión. El grupo de edad seleccionado fue niños y niñas de 6 a 12 años en base a programas de prevención y para promover la salud bucal, cuyo enfoque actual es el preescolar (menores de 6 años) para que la próxima etapa de 6 a 12 años ${ }^{17}$ pueda reflejar el estado de salud bucal. En su mayoría, la población infantil correspondía a niños que asistían de segundo al sexto año de educación básica, equivalente al 83,9\% de todos los alumnos del establecimiento, considerando que 4 niveles no se incluyeron en este estudio. Se determinó su riesgo de caries mediante CAST modificado.

El protocolo de muestreo y consentimiento informado, fueron certificados por el Comité de Bioética de la Universidad de Talca.

La muestra de saliva no estimulada en ayunas $^{18}$ se colectó en tubos Eppendorf de 1,5 ml (Globe Scientific-USA), los criterios de inclusión fueron: no cepillarse, no haber consumido antibióticos durante los últimos 3 meses y si los padres firmaron el consentimiento y los niños confirmaron el acuerdo. Luego, la muestra se transportó a $4^{\circ} \mathrm{C}$ en un contenedor conservador acompañado al Laboratorio de Investigación Microbiológica en la Universidad de Talca para su procesamiento inmediato.

Procesamiento de la muestra.

Las muestras de saliva fueron homogenizadas con la ayuda de un agitador de tubos (Tipo 37600 Maxi Mixer Mix II, Panel, EE. UU. - S.A.) durante 30 segundos. Posteriormente, se agregaron 
1 ul de la muestra a $999 \mathrm{ml}$ de agua destilada contenida en un tubo Eppendorf estéril de 1,5 ml (Globe Scientific USA), esta solución se homogeneizó de nuevo durante 30 segundos y se tomó la dilución final de 50 ul. el medio TYCSB (tripticasalevadura-cistina-sacarosa-bacitracina) ${ }^{19}$ otra fracción de la muestra se sembró en caldo MRS (Merck, Alemania), selectivo para Lactobacillus. ${ }^{20}$ Estas placas se incubaron en jarras anaeróbicas Gas-Pak (Biomerieux, Francia) a $37^{\circ} \mathrm{C}$, durante 48 horas en una estufa Pasteur (Memmert 300 GrimbH-Co.KG-Alemania).

Identificación y selección de cepas de Lactobacillus spp. y Streptococcus mutans.

Lactobacillus. Después del tiempo de incubación, se seleccionaron 5 colonias aleatorias de cada placa. Se registraron las características morfológicas de las colonias como el color, tamaño ( $\mathrm{mm})$, la forma y la forma, haciendo referencia a las propiedades enumeradas en el Manual de Bergey. ${ }^{21}$ Se realizaron tinciones de Gram en todas las colonias. Luego se hicieron tres pasajes sucesivos en caldo MRS para estabilizar los aislamientos.

Streptococcus mutans. Las colonias de Streptococcus mutans se analizaron macroscópicamente observando un grano con forma de carácter y una textura de azúcar, de bordes blanquecinos, firmes $y$ adherentes. ${ }^{22}$ Posteriormente, se clasificaron en dos grupos: aquellos que producen y no producen glicocalix. En medio sólido, las cepas productoras de glicocálix que forman colonias circundantemente presentan una masa viscosa clara de material transparente. Las cepas no productoras de glicocálix forman colonias que carecen de este material viscoso. ${ }^{23}$
El crecimiento bacteriano obtenido a partir de muestras procesadas por el método cuantitativo se contó a ojo.

El recuento total de colonias de Lactobacillus spp. presentes en la placa de Petri se multiplicaron por el factor de dilución (103) y se expresaron en unidades formadoras de colonias por $\mathrm{ml}$ de saliva $(\mathrm{CFU} / \mathrm{ml}){ }^{24}$

Ensayos de inhibición del crecimiento bacteriano.

Agar doble capa. Se usó una placa de agar doble de prueba para estudiar el efecto antimicrobiano de Lactobacillus spp. Los inóculos de aislamientos clínicos se transfirieron a caldo MRS y se incubaron a $37^{\circ} \mathrm{C}$ durante 48 horas. Posteriormente, se sembraron $20 \mu \mathrm{l}$ de líquido de cultivo se sembraron en césped en agar MRS (MerckAlemania). El ensayo de doble placa consiste en agar de dos capas, el agar MRS contiene el primer desarrollo de colonias de Lactobacillus aisladas. Sobre las colonias se vierte una mezcla de $S$. mutans con agar BHI semisólido $(0,8 \%)$ y posteriormente la placa se incuba en ambiente anaeróbico a $3^{\circ} \mathrm{C} \mathrm{C}$ durante toda la noche para permitir el crecimiento de $S$. mutans. Finalmente se observaron halos inhibitorios alrededor de las colonias de Lactobacillus. ${ }^{25}$

Técnica de sobrecrecimiento. Se realizó la siembra de una gota de caldo de cultivo MRS (Merck-Germany) que contenía Lactobacillus spp. en una placa con Agar BHI (Merck Germany) $+1 \%$ de dextrosa (Merck Germany) y después de 48 horas de incubación a $37^{\circ}$ en jarras Gas Pak de anaerobiosis (Biomerieux, Francia) se observó sobrecrecimiento bacteriano el cual fue eliminado con una tórula estéril 
que contenía alcohol desnaturalizado al $96 \%$ (Reutter-Santiago, Chile). Luego sobre sobre esta placa se sembró en césped $50 \mu \mathrm{l}$. de Streptococcus mutans y se incubo por 48 horas en ambiente anaeróbico e incubadas a $37^{\circ} \mathrm{C}$, durante toda la noche.

Análisis de la sustancia enzimática antagonista producida por Lactobacillus.

Sensibilidad a la catalasa. Se filtró $1 \mathrm{ml}$ del sobrenadante de un cultivo bacteriano de Lactobacillus a través de filtros de membrana con un tamaño de poro de 0,45 $\mu \mathrm{m}$ (Millipore) colectándose en un tubo Eppendorf estéril. El filtrado se trató con 100 ul de una solución estéril de catalasa (Sigma-Aldrich). Tanto el cultivo como el filtrado se homogeneizaron en Vortex Monomixer (Sarsted-Alemania) para tubos Eppendorf. Posteriormente, se llevó a cabo un ensayo de antagonismo biológico, utilizando como bacteria blanco a Streptococcus mutans. La prueba se realizó mediante técnica de pocillo en agar y se incubo por 48 horas en jarras de anaerobiosis. La prueba de sensibilidad a catalasa se aplica para descartar que la actividad inhibitoria sea debido a la producción de peróxido de hidrógeno. ${ }^{26}$

Sensibilidad a enzimas proteolíticas. El sobrenadante antibacteriano se sometió a tratamiento con las siguientes enzimas proteolíticas: tripsina, quimotripsina, proteasa, lisozima, proteinasa K (SigmaAldrich) a una concentración final de $1 \mathrm{mg} / \mathrm{ml}$ de sobrenadante. Posteriormente, para analizar el resultado de esta prueba se realizó la misma prueba biológica descrita anteriormente. Con las enzimas proteolíticas se pretende averiguar la naturaleza proteica de la sustancia antagónica con el propósito de asociarla a una bacteriocina.
Estabilidad de la sustancia antagonista frente a las propiedades físicas.

Estabilidad térmica. El sobrenadante antibacteriano se incubó a $4{ }^{\circ} \mathrm{C}-72{ }^{\circ} \mathrm{C}$ y $37^{\circ} \mathrm{C}$ durante 5 días y $80{ }^{\circ} \mathrm{C}, 100{ }^{\circ} \mathrm{C}$ y $121{ }^{\circ} \mathrm{C}$ durante 15 minutos. Después de los períodos de incubación, la actividad antibacteriana de los sobrenadantes tratados se determinó utilizando la técnica de difusión de pocillos, como se describe anteriormente. Para $4^{\circ} \mathrm{C} y$ $-72^{\circ} \mathrm{C}$ se utilizaron refrigeradores fijados a estas temperaturas; Horno Pasteur a $37^{\circ} \mathrm{C}$ (Memmert 300 GrimbH-Co.KGAlemania); para temperaturas de $80{ }^{\circ} \mathrm{C}$ y $100{ }^{\circ} \mathrm{C}$ un precipitado de $250 \mathrm{ml}$ de vaso de vidrio que contiene $180 \mathrm{ml}$ de agua destilada colocada en un trípode con rejilla metálica y expuesta a una llama del quemador Bunsen a la temperatura que se midió con un termómetro de mercurio para uso de laboratorio y finalmente el sobrenadante a $121^{\circ} \mathrm{C}$ se llevó en autoclave (Orthman-USA). ${ }^{27}$

Estabilidad del pH. El sobrenadante antibacteriano se sometió a tratamiento con $\mathrm{HCl}$ y $\mathrm{NaOH}$ concentración $1 \mathrm{M}$ tanto al $\mathrm{pH}$ apropiado. Los siguientes $\mathrm{pH} 1,4,6$, $7,8,10$ se analizaron, ya que se estableció un control de $\mathrm{pH} 7$ para determinar que la actividad inhibitoria se debe a un $\mathrm{pH}$ ácido o básico. Posteriormente, se realizaron ensayos de antagonismo biológico como se describió anteriormente. ${ }^{26}$

Identificación de la especie de Lactobacillus.

La identificación de la cepa de producción de Lactobacillus se realizó utilizando la prueba Api $50 \mathrm{CH}$, de acuerdo con las instrucciones del fabricante. 
Análisis estadístico

Los resultados se analizaron mediante tablas de contingencia y ANOVA multivariado, interpretados para el Graph Pad Prism software versión 6 y Microsoft Office Excel 2013. Para este último cálculo se realizó una proporción (porcentaje) basada en el número de cepas de Lactobacillus con recuento de actividad y el promedio según a la fórmula:

$$
\begin{aligned}
& \mathrm{R} \rightarrow 100 \% \\
& \mathrm{~N} \rightarrow \mathrm{X} \\
& \text { Entonces: } \quad \mathrm{X}=\frac{\mathrm{N} \times 100}{\mathrm{R}}
\end{aligned}
$$

Donde:

$\mathrm{R}=$ Media recuento de Lactobacillus

$\mathrm{N}=$ (número de personas con Lactobacillus con actividad)

\section{Resultados}

\section{Crecimiento y aislamiento}

A partir de muestras de saliva, se cultivó TYCSB en agar y crecimiento en agar MRS de Streptococcus mutans y Lactobacillus spp., respectivamente.

Pruebas de inhibición por agar de doble capa y utilizando el método de sobrecrecimiento resultaron positivos.

Fueron verificadas las zonas de inhibición utilizando el agar de doble capa de prueba que confirma la presencia de una sustancia antagonista producida por varias colonias de Lactobacillus spp. en Streptococcus mutans. También se observan halos inhibitorios de diferente tamaño.

En la técnica de pocillo el producto antagónico de la cepa Lactobacillus Z2 (mayor inhibición) genero un halo inhibitorio que explica la inhibición del crecimiento de Streptococcus mutans mostrada. Se destaca que la cepa Lactobacillus Z2 produce una sustancia antagónica que genera el halo inhibitorio de mayor diámetro.

También se observó una inhibición del crecimiento bacteriano mediante el método de sobrecrecimiento en el que la cepa Lactobacillus Z2 después de eliminar el crecimiento es una sustancia antagonista en el medio de cultivo, volvió a generar la inhibición de Streptococcus mutans comprobando mediante 3 métodos una acción de una bacteriocina (proteína enzimática).

La catalasa no actuó sobre la sustancia de la cepa antagonista de Lactobacillus Z2 manteniéndo sin cambios su actividad antibacteriana contra Streptococcus mutans (datos no revelados). Además, la actividad de las enzimas proteolíticas inhibió la acción antibacteriana de la sustancia biológica antagonista. La sustancia antagonista mostró una tolerancia interesante a todas las temperaturas estudiadas confirmadas por el mantenimiento de la propiedad inhibidora antibacteriana. Con respecto a la acción se observó una óptima acción a $\mathrm{pH}$ 4 y 7 . No se observó actividad antagónica inhibitoria con los otros $\mathrm{pH}$ analizados (Datos no mostrados). (Figura 1)

Se demostró que niños participantes del estudio presentan Lactobacillus que producen sustancias antagónicas sobre Streptococcus mutans de los cuales más del $50 \%$ de ellos tienen en su microbiota oral Lactobacillus capaces de sintetizar productos antagónicos contra Streptococcus mutans. Fue interesante observar que la mayor parte de los Lactobacillus que 

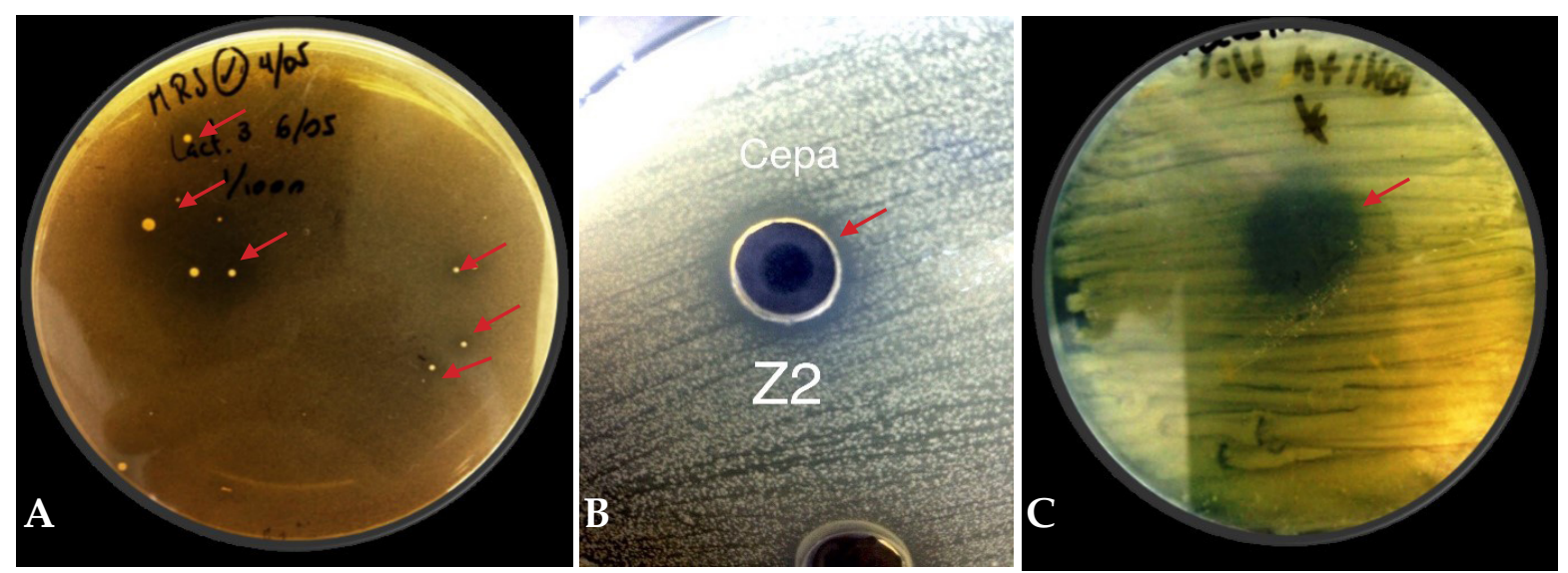

Figura 1. Inhibición. En A, obsérvese flechas rojas que indican zonas oscuras áreas de no crecimiento del cesped de Streptococcus mutans sin glicocálix. B. Centrifugado del caldo colocado en un pocillo muestra la inhibición frente a Streptococcus mutans con Glicocalix. C. Técnica de sobrecrecimiento muestra la zona de donde se difunde la sustancia antimicrobiana la misma que impide el crecimiento de Streptococcus mutans.

demuestran capacidad de producir sustancias antagónicas pertenecen al grupo de niños libres de caries pero también está presente esta probable bacteriocina en niños con caries.

El $63 \%$ de los niños estudiados presenta en saliva Lactobacillus sin capacidad antagónica respecto del $37 \%$ cuyas cepas de Lactobacillus tienen actividad antagónica sobre Streptococcus mutans. El $1 \%$ de estas cepas pertenece al grupo con caries, $11 \%$ al grupo sin caries activa y el $25 \%$ al grupo libre de caries. (Figura 2)

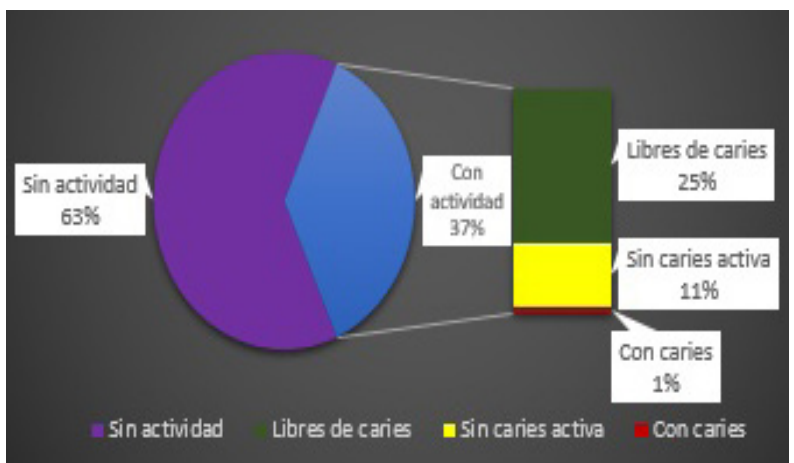

Figura 2. Relación entre presencia de Lactobacillus $y$ caries.
Los recuentos de Streptococcus mutans de los tres grupos se halló una diferencia significativa según el riesgo de caries de los participantes. (Figura 3)

El test Api $50 \mathrm{CH}$ permitió identificar a la cepa de Lactobacillus Z2, mayor cepa productora de sustancia antagónica (dato no mostrado), como Lactobacillus fermentum.

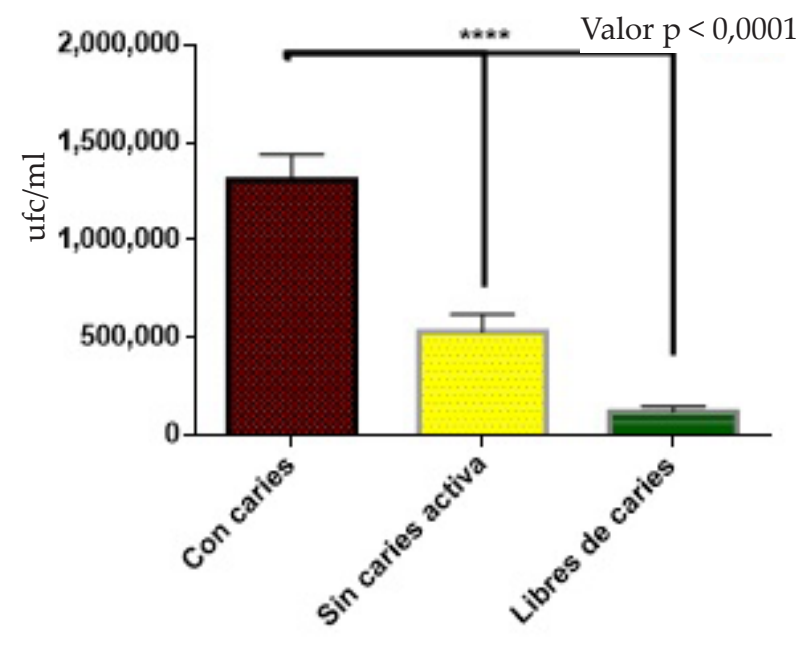

Figura 3. Recuento de Streptococcus mutans según riesgo de caries. 


\section{Discusión}

Esta investigación demuestra que los niños participantes del estudio presentan Lactobacillus que producen sustancias antagónicas sobre Streptococcus mutans de los cuales más del $50 \%$ de ellos tienen en su microbiota oral Lactobacillus capaces de sintetizar productos antagónicos contra Streptococcus mutans. Fue interesante observar que la mayor parte de los Lactobacillus que demuestran capacidad de producir sustancias antagónicas pertenecen al grupo de niños libres de caries pero también está presente esta probable bacteriocina en niños con caries aunque en menor proporción, al respecto varios estudios apoyan los presentes resultados. ${ }^{28-30}$ Los niños que tenían bajo riesgo de caries presentaron mayor cantidad de Lactobacillus el cual producía la sustancia antagónica contra Streptococcus mutans disminuyendo el recuento del mismo así creemos también puede suceder in vivo como lo muestra un estudio clínico, ${ }^{31}$ el resto de los niños, sin embargo, no está libre de la presencia de estas especies protectoras esta variación puede deberse a una modificación de su ecosistema bucal. ${ }^{32}$ Hoy podemos ya pensar en el uso de estas especies que puedan controlar a bacterias cariogénicas pues contamos con evidencia al respecto, ${ }^{33}$ y así los probióticos orales podrían convertirse en una terapia biológica activa a corto plazo..$^{34,35}$

\section{Conclusiones}

De acuerdo con los resultados obtenidos en esta investigación, la presencia de un mayor porcentaje de cepas Lactobacillus fermentum corresponde a la microbiota oral de niños sin caries y caries inactivas. Este resultado particular puede argumentar que la capacidad potencial de control biológico se ejerce sobre las bacterias $S$. mutans se verifica qué contó los $S$. mutans de la misma muestra de saliva estudiada donde se detectan la mayoría de las bacterias cariogénicas en niños con caries activas y menos niños sin caries.

Lactobacillus fermentum pertenece al grupo de las Bacterias Ácido Lácticas y permitiria una consideración seria posterior para su uso como probiótico en el control de estudios de antagonismo. Por lo cual estudios posteriores abren un umbral de investigación necesaria que promoveria el conocimiento de la naturaleza química a nivel molecular de este nuevo producto y también investiguen su codificación genética para abordar el problema desde un punto de vista biotecnológico.

\section{Agradecimientos}

Al Departamento de Microbiologia de la Universidad de Talca, a la Agencia de Cooperación Internacional para el Desarrollo del Gobierno de Chile.

\section{Referencias bibliográficas}

1. Petersen PE. Global policy for improvement of oral health in the 21st century implications to oral health research of World Health Assembly 2007, World Health Organization. Community Dent Oral Epidemiol. 2009; 37 (1): 1-8.

2. Simón-Soro A. Solving the etiology of dental caries. Trends Microbiol. 2015;23:2-76.

3. $\mathrm{Xu} \mathrm{X}$. Oral cavity contains distinct niches with dynamic microbial communities. Environ Microbiol. 2015; 17(3): 699-71 
4. Marsh PD. Are dental diseases examples of ecological catastrophes? Microbiology. 2003; 14: 279-94.

5. Clarke JK. On the bacterial factor in the aetiology of dental caries. Br J Exp Pathol. 1924; 5(3): 141-147.

6. Li Y. Characterizing Diversity of Lactobacilli Associated with Severe Early Childhood Caries: A Study Protocol. Adv Microbiol. 1; 5(1): 9-20.

7. Graciano ME, Correa YA, Martínez CM, Burgos A, Ceballos JI, Sánchez LF. Streptococcus mutans y caries dental en América Latina. Revisión sistemática de la literatura. Rev Nac Odontol. 2014; 8 (14): 32-45.

8. Teanpaisan R, Piwat S, Tianviwat S, Sophatha B, Kampoo T. Effect of Long-Term Consumption of Lactobacillus paracasei SD1 on Reducing Mutans streptococci and Caries Risk: A Randomized PlaceboControlled Trial. Dentistry Journal. 2015; 3(2): 43-54.

9. Camelo-Castillo A, Benítez-Páez A, Belda-Ferre P, Cabrera-Rubio R, Mira A. Streptococcus dentisani sp. nov., a novel member of the mitis group. Int J Syst Evol Microbiol. 2014; 64(Pt 1):60-5.

10. Twetman S. Probiotics for caries prevention and control. Adv Dent Res. 2012; 24(2): 98-102.

11. Güngör öE, Kırzıoğlu Z, Dinçer E, Kıvanç M. Who will win the race in childrens' oral cavities? Streptococcus mutans or beneficial lactic acid bacteria? Beneficial microbes. 2013; 4(3):237-45.

12. Chuang L-C. Probiotic Lactobacillus paracasei effect on cariogenic bacterial flora. Clin Oral Investig. 2011; 15(4):471-6.

13. Caufield PW. Oral Lactobacilli and Dental Caries: A Model for Niche Adaptation in Humans. J Dent Res. 2015 ; 94(9 Suppl):110S-8S.

14. Piwat S. Lactobacillus species and genotypes associated with dental caries in Thai preschool children. Mol Oral Microbiol. 2010; 25(2): 157-64.

15. Yang R. Determining the genetic diversity of lactobacilli from the oral cavity. J Microbiol Methods. 2010; 82(2): 163-169.

16. Byun R. Quantitative analysis of diverse Lactobacillus species present in advanced dental caries. J Clin Microbiol. 2004; 42(7): 3128-3136.

17. Cornejo-Ovalle M, Fajreldin V, Werlinger F, Candia O, Cruces G, Farfán J, et al. Mesas de trabajo intersectorial y política de salud oral enfocada a la niñez. Rev Clin Periodoncia Implantol Rehabil Oral. 2015; 8 (3): 261-2.

18. Ilave MG, Fernández LO, Calderón KM, Villacampa SC. Eficacia de una medida preventiva para el niño con riesgo cariogénico asociada a la estabilidad de pH salival. Odontol Sanmarquina. 2007;10.1:25-7.

19. Lobos O. In vitro antimicrobial effect of bacteriocin PsVP-10 in combination with chlorhexidine and triclosan against Streptococcus mutans and Streptococcus sobrinus strains. Arch Oral Biol. 2009; 54 (3): 230.

20. Abo-Amer AE, Shobrak MY. Partial characterization of a bacteriocin produced by Lactobacillus salivarius isolated from oral cavity of desert foxes. Afr J Microbiol Res. 2012; 6: 6589-99.

21. Goodfellow, Michael, Fred A. Rainey, and Karl-Heinz Schleifer. Bergey's Manual ${ }^{\circledR}$ of Systematic Bacteriology: Volume Two The Proteobacteria Part B The Gammaproteobacteria. Springer, 2005.

22. Ojeda-Garcés JC, Oviedo-García E, Salas LA. Streptococcus mutans y caries dental. CES Odontología. 2013; 26 (1): 44-56.

23. De la Higuera A. In-vitro susceptibility, tolerance and glycocalyx production in Streptococcus mutans. J Antimicrob Chemother. 1997; 40 (3): 359-63.

24. Gomez Parada PA, Vives Contardo J. Determinacion de riesgo cariogenico utilizando el cuadro de riesgo de la ficha de odontopediatria y el programa computacional Cariogram, Universidad de Talca, 2008: Estudio piloto.

25. Hassan YI. Antifungal activity of Lactobacillus paracasei ssp. tolerans isolated from a sourdough bread culture. Int J Food Microbiol. 2008; 121(1):112-5

26. Busarcevic M, Dalgalarrondo M. Purification and genetic characterisation of the novel bacteriocin LS2 produced by the human oral strain Lactobacillus salivarius BGHO1. J Antimicrob Agents. 2012; 40(2): 12734.

27. Wannun P. Purification and characterization of bacteriocin produced by oral Lactobacillus paracasei SD1. Anaerobe. 2014; 27: 17.

28. Rebolledo, M.; Rojas, E.; Salgado, F. Efecto de dos probioticos que contienen cepas de Lactobacillus casei variedad rhamnosus y Lactobacillus johnsonii sobre el crecimiento in vitro de Streptococcus mutans. Int. J. Odontostomat. 2013; 7 (3); 415-419. 
29. Kõll-Klais P, Mändar R, Leibur E, Marcotte H, Hammarström L, Mikelsaar M. Oral lactobacilli in chronic periodontitis and periodontal health: species composition and antimicrobial activity. Oral Microbiol Immunol. 2005; 20 (6): 354-361.

30. Baca-Castañón ML, De la Garza-Ramos MA, Alcázar-Pizaña AG, Grondin Y, Coronado-Mendoza A, Sánchez-Najera RI, Cárdenas-Estrada E, Medina-De la Garza CE, Escamilla-García E. Antimicrobial effect of Lactobacillus reuteri on cariogenic bacteria Streptococcus gordonii, Streptococcus mutans, and periodontal diseases Actinomyces naeslundii and Tannerella forsythia Probiotics Antimicrob Proteins. 2015; 7 (1): 1-8.

31. Teanpaisan, Rawee, et al. Effect of long-term consumption of Lactobacillus paracasei SD1 on reducing mutans streptococci and caries risk: a randomized placebo-controlled trial. Dent J. 2015; 3(2): 43-54.

32. Bustillos Torrez W, Lobos Gilabert O, Padilla Espinoza C, Brevis Azócar P. Lactobacillus presentes en la cavidad oral de infantes con bajo riesgo cariogénico producen sustancias antagónicas activas contra Streptococcus mutans; 2016.

33. Fernández CE, Giacaman RA, Tenuta LM, Cury JA. Effect of the probiotic Lactobacillus rhamnosus LB21 on the cariogenicity of Streptococcus mutans UA159 in a dual-species biofilm model. Caries Res 2015; 49 (6): 583-590.

34. López-López A, Camelo-Castillo A, Ferrer MD, Simon-Soro A, Alex Mira A. Health-associated niche inhabitants as oral probiotics: the case of Streptococcus dentisani. Front. Microbiol. 2017; 8: 379.

Recibido: 21/10/18

Aceptado: 28/06/19

Correspondencia: Willy Bustillos Torrez, e-mail: wbustillos@utalca.cl. 